International Journal of Distributed and Parallel Systems (IJDPS) Vol.3, No.1, January 2012

\title{
Energy Efficient, Scalable and Reliable MAC Protocol for Electromagnetic Communication among Nano Devices
}

\author{
${ }^{1}$ V.Srikanth, ${ }^{2}$ Sindhu Chaluvadi, ${ }^{3}$ Sandeep, ${ }^{4}$ Vani, ${ }^{5}$ Venkatesh \\ ${ }^{1}$ Head of the Department, Information Science and Technology, K L University, \\ Andhra Pradesh \\ srikanth_isteklce.ac.in \\ Students, Department Of Information Science And Technology, K L University

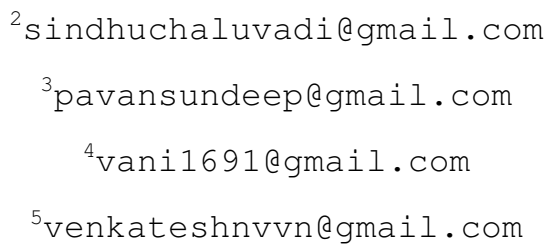

Abstract

The possibility of manipulating and assembling objects at the atomic scale has paved way for a future generation of computing machines, where nanoscale devices substitute silicon-based transistors. In this paper we review the fundamental issues arising when nano scale devices communicate in a dense employed network. We describe the existing modes of communication among the nano devices with an emphasis on Electromagnetic Communication. As a solution, an Energy Efficient, Scalable and Reliable MAC Protocol has been proposed along with the structures of control packet and data packet, thereby constrained to certain open challenges.

\section{Keywords}

Nanonetwork, electromagnetic, clustering, master node, control packet.

\section{Introduction}

With the growing edge over nanotechnology several tools have been developed to enhance communication among the nanodevices(defined later). The improvements made in this field have shown a spectacular result in various applications such as technology, medicine etc. Though this novel approach is being applied widely in the recent times little attention has been paid, so far, in understanding communication at the nanoscale level. Nanodevices are the basic functional unit capable of performing minute tasks like computation, actuation and sensing.

DOI : $10.5121 /$ ijdps.2012.3121 
International Journal of Distributed and Parallel Systems (IJDPS) Vol.3, No.1, January 2012

They are typically of the size of nanometer[3]. Nanonetworks are collection of these devices to coordinate these devices and also to enable communication among these devices [3]

\section{Modes of Nanonetworking:}

The communication among nanomachines is specifically carried through the below mentioned four types: nanomechanical, acoustic, electromagnetic, and chemical or molecular[2]. Nanomechanical Communication happens in a way where there is mechanical contact among the devices through some hard wire junctions and is not applicable in all environments. In acoustic communication happens with the appliance of pressure on sound waves. In electromagnetic communication, information is transmitted through modulated electromagnetic waves. The rest of the paper focuses on this way of communication among nanodevices using this technique. Lastly, in molecular communication, first introduced in [3], the message is encoded using molecules. Molecular transceivers are able to react to specific molecules, and to release others in response to an internal command. In creating molecular communicating systems, we use existing biological nano-scale communication mechanisms .

\section{Electro Magnetic Communication}

Due to some peculiar properties shown by graphene based materials communication through electromagnetic means became the most demanding type. In detail, Graphene is a one-atomthick planar sheet of carbon atoms which are bound in a honeycomb structure. The wave propagation inside Graphene structures has been proved to be hundreds of times lower than the speed of light [5]. This new paradigm requires transmission mechanisms that make a reasonable usage of the available energy. Besides, nanodevices exhibit some limitations due to their small size. Thus, nano-devices require low complex and energy efficient communications schemes.

Despite all these disadvantages that arise from the fact of scaling down devices, Graphenebased nanoscale devices promise high switching frequencies due to the low electron scattering in nanoscale Graphene structures[6]. Hence, we envision that electronic nano-devices might be simple and energy-constrained, but provide fast[4] communication.

\section{MAC Issues}

Nanonetworks are supposed to be densely populated networks. A high number of nodes are required to maintain the network connectivity and to have a macroscale effect from the millions of nano-effects. However, the number of nodes per square meter will depend, amongst other factors, on the final network application.

Moreover, nano-devices are severely energy-constrained machines since their dimensions make challenging to store or harvest energy. Therefore, not only new energy nano-harvesting systems

are required, but the new tailored transmission schemes should also consider those limitations to introduce more energy-efficient schemes. 
Due to the high number of nodes and the mentioned energy constraints, we foresee the necessity of organizing the access to this shared medium. Molecular and electromagnetic-based communications need to find a novel way to schedule multiple transmissions in time, frequency or code. This means that new energy-efficient complexity-aware Medium Access Controls (MACs)[4] need to be build up from the physical consideration that governs the nanoscale peculiarities. Here we propose a novel multiple access method derived from an existing transmission scheme for electromagnetic communications, that intends to mitigate multi-user interference than completely avoid it

\section{Proposed MAC Protocol}

This protocol can be divided in to two main stages: the Selection of Master Node and the Data transmission.

\subsection{Stage I :}

Initially we assume that the nanonodes combine to form a network. A master node is selected among the available nodes, which is equidistant from the rest of the nodes.This metric helps remove ambiguity that were in the conventional mechanisms and lessen the collision possibility as well.

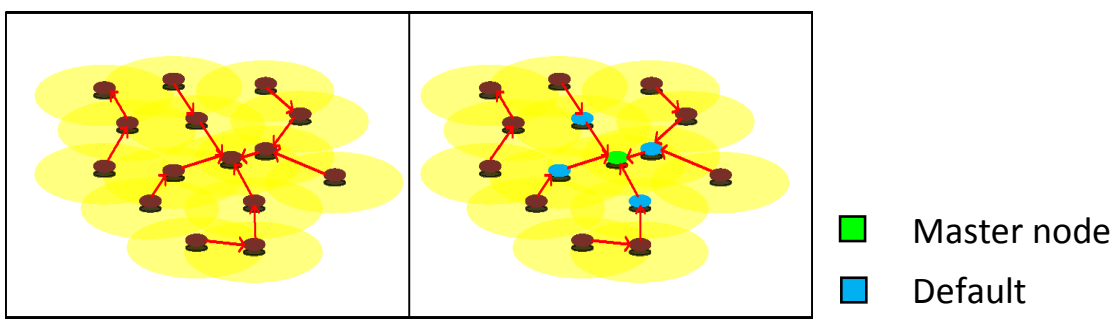

Fig.1.Selection of Master Node

The selection of the node $\mathrm{m}$ must be announced to the other nodes so as to ignite the communication.The nodes are now equally distributed and each tries to send its packets to M. It is the responsibility of the node $\mathrm{M}$ to allocate the channel to the requested device for a certain period of time, based on the priorities of the devices or the urgency of the data that it has to transfer. However,this method works iff the number of nodes in the scenario fall within 50 .

If the number exceeds 50,then an alternative method is applied where the nanodevices are divided into clusters.In order to support data aggregation through efficient network organization, nodes can be partitioned into a number of small groups called clusters. Each cluster has a coordinator, referred to as a cluster head $(\mathrm{CH})$, and a number of member nodes.

Clustering results in a two-tier hierarchy in which $\mathrm{CHs}$ form the higher tier while member nodes form the lower tier[7] . Fig .2 illustrates data flow in a clustered network. The member nodes report their data to the respective $\mathrm{CHs}$. The $\mathrm{CHs}$ aggregate the data and send them to the central base through other $\mathrm{CHs}$. Because $\mathrm{CHs}$ often transmit data over longer distances, they 
lose more energy compared to member nodes. The network may be reclustered periodically in order to select energy-abundant nodes to serve as $\mathrm{CHs}$, thus distributing the load uniformly on all the nodes. Besides achieving energy efficiency, clustering reduces channel contention. Hence, periodic reclustering is necessary in order to heal disconnected regions and distribute energy consumption across all nodes. Periodic reclustering is also necessary, as the parameters used for clustering (e.g., the remaining energy, node degree, etc.) are dynamic, and packet collisions, resulting in better network throughput under high load. In this scenario, there are two types of communication possible :

- Intra Cluster and

- Inter Cluster

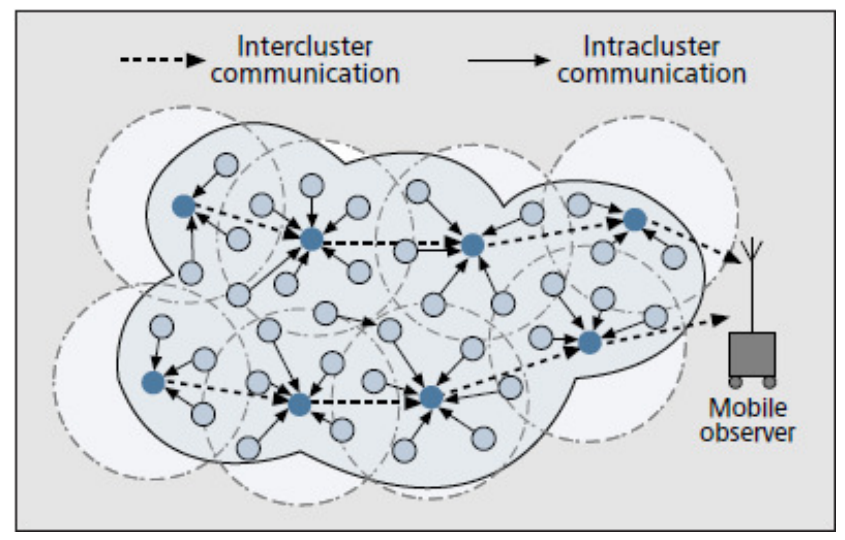

Fig.2.Clustered NanoDevices

Scheduling intracluster and intercluster transmissions is another important design challenge[7]. Since clustering is typically employed in applications where data aggregation is performed, time division multiple access (TDMA) is best for intracluster transmissions. This is because a $\mathrm{CH}$ can set the TDMA schedule and inform its cluster members about it. The problem is how to prevent the TDMA intracluster transmissions from colliding with transmissions in neighboring clusters or with intercluster frames, especially when $\mathrm{CHs}$ use longer ranges for communicating with each other.

After the selection of the master node, this information is passed by broadcasting a TDMA frame to all the nodes in the network.

Here raises another scenario where few other nodes are to be added in to the network. If this happens the selection of master node is to be rescheduled by iterating the above procedure. Since the master node is repeatedly subjected to change whenever new nodes are added to the network, energy dissipation is reduced.

The number of nodes to be added is also a considerable constraint to achieve scalability. If the number of additional nodes is less than 10 then the above mentioned technique can be applied. 
If the number exceeds 10 then again we have to roll back to the Clustering mechanism which is described in detail in the above sections.

\subsection{Stage II :}

This stage is concerned with the communication of other devices with the node M. Any node that requires the channel sends a control packet with fields containing destination address, source address, priority bit, to the node $\mathrm{m}$. Priority bit number is a single bit field that represents the priority of the device. If the bit is set to 0 then the device can be scheduled sequentially. If the bit is 1 , then the device has an emergency channel requirement.

Scheduling of these devices is done through queuing at the master node by using Round Robin algorithm(that assumes all the devices of equal priority). After queuing, M starts allocating the channel on the FCFS basis. In some cases, if a node is to be allocated with the channel immediately then it sets it priority bit to 1 and hence the requested channel is allocated.

If $\mathrm{MN}$ wishes to grant the channel to the node $\mathrm{A}$, it first sends a REQUEST signal to the receiver $\mathrm{B}$. If the receiver is free, then it sends an acknowledgement (ACK) signal to $\mathrm{MN}$ allowing the communication. MN in turn sends the same ACK signal to the requested device A. Hence the sender is allocated with the channel and the transmission of the packet takes place. After receiving all the packets $\mathrm{B}$ sends the acknowledgement to the master node indicating that the transfer is successful .

In order to avoid collisions between the data packets and ACKs, the sender mentions the packet count in the first packet and receives an ACK from MN after all the packets arrive at the receiver. If the ACK is not received at the sender node within a stipulated time, indicating an error in transmission then all the packets are resent. Since the channel is allocated for transmission between two devices there will be no collisions from other nodes and hence reliability is achieved. 


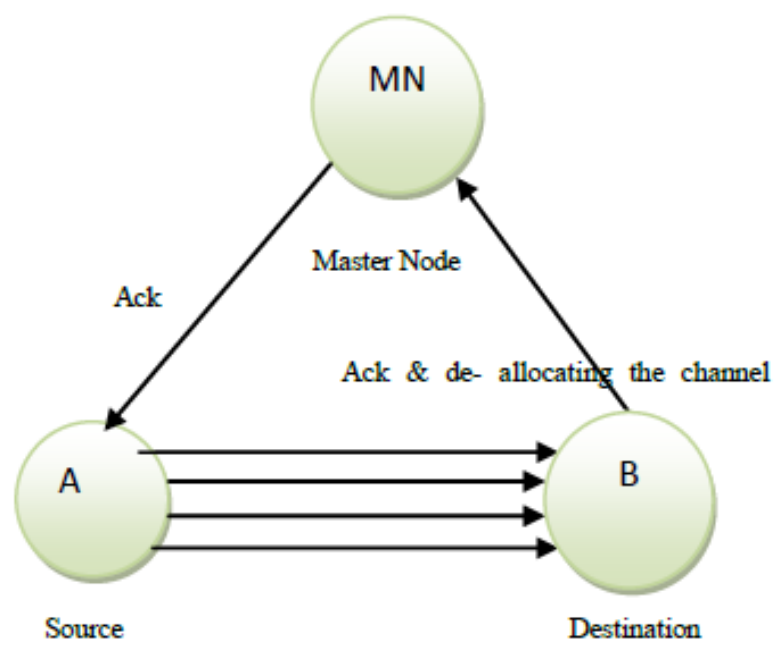

Fig.3. Transmission of packet

Energy Efficiency can be taken as a measure of the extent to which collisions are reduced. Since there are no collisions, the energy required to retransmit the packets can be eliminated totally. The other area where energy is conserved is selecting an equidistant method for the selection of master node all the nodes will require equal amount of energy to transmit the control packet. Whenever the nodes are added in to the network the master node is changed where energy dissipation is reduced. Considering all these factors energy will be saved or consumption of energy is reduced to a very great extent.

\subsection{Packet Structures:}

Two types of Packet structures are involved here: Control Packet, Data Packet.

\subsubsection{Control Packet}

Control Packet is a dummy packet, free of the data, that helps in allocation of the channel to the requested device. The fields included in its structure are Destination Address, Source Address, Priority bit and Synchronization trail.

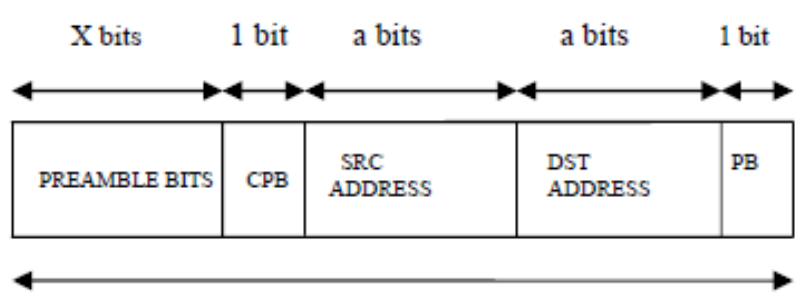

Fig.4.Control packet 
The preamble bits present in the packet are used for the synchronization of the nanomachines to enable communication. The conflict arises as to which address has to be mentioned in the DST ADDRESS field. Is it the master node address or the destination node address?. To resolve this we include another field known as the CPB is the Control packet bit which distinguishes the control packet from the data packet. If the packet is a control packet then the bit CPB is set to 1, otherwise the packet is taken to be a data packet. Now the packet whose bit is set to 1 will be sent to the master node thereby requesting the channel.

\subsubsection{Data Packet}

Data packet structure represents the original data to be transferred with the following form. The structure consists of a trail part and the data part. The data to be transferred is encoded in the data field and then transmitted.

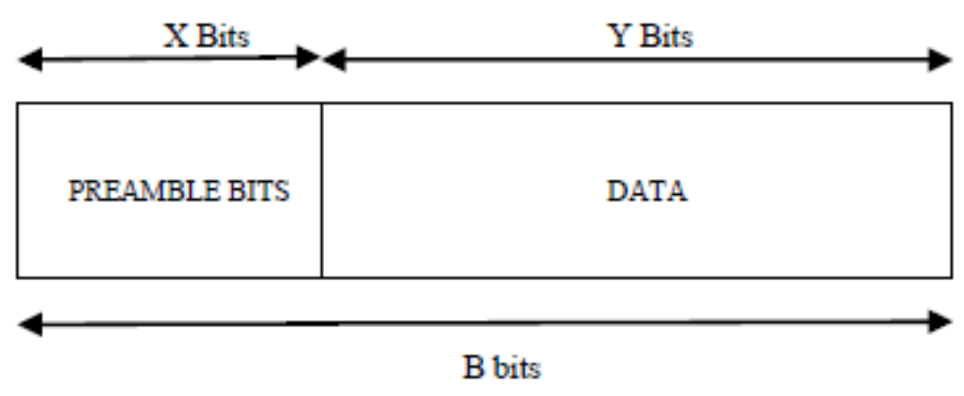

Fig.5.Data Packet

\subsection{Algorithm}

A brief description of the above mentioned procedure is given below in the form of an algorithm:

1. A Nanonetwork is formed with ' $n$ ' no. of nanonodes in it.

2. Election of the master node is done based on the value of $n$. If $n<50$ then we use EQUIDISTANT metric method.

3. If $n>50$ then clustering is applied. Protocols for intercluster and intracluster communications like HEED are employed for the selection of master node.

4. Now the nanodevice requests the master node to allocate the channel.

5. The nanodevice will send a control packet to the master node. The master node will know that it is a control packet by setting the CPB to 1 .

6. Queuing is done at the master node which consists of all the nanodevices that request the allocation of channel. The queue scheduling followed here is Round Robin Scheduling.

7. If a nanodevice needs the channel prior to all then the PB bit will be set to 1 .

8. After queuing the devices the first node in the queue will be allocated with the channel. 
International Journal of Distributed and Parallel Systems (IJDPS) Vol.3, No.1, January 2012

9. Now the channel is used only by the two devices that have been mentioned in the control packet.

10. After the data transmission is completed the master node takes back the control of the channel and reallocates it to the next device on the queue.

\section{Conclusions}

Nanonetworks are not only downscaled versions of the classical macroscale networks, but networks with new peculiar requirements. The nanoscopic dimensions of their nodes as well as their use in unusual scenarios demand for novel communication paradigms that enable the interconnection of nano-nodes in any nanoscale environment.

We also introduced a new MAC protocol tailored to the underlying physical layer though emphasis has not been made on the physical layer concepts. However, this protocol has only been described and numerically evaluated, but a reliable simulation analysis is still missing. Because of this, we propose to evaluate the protocol performances through a network simulator, such as NS-2.

Finally, we have been saying that nano-devices have limited energy storage capabilities and they necessarily have to harvest energy from their surroundings. However, energy models for nano-devices are still missing and hence protocol evaluations are not enough accurate. Therefore, the community needs to only cope with reliable mechanism for communication.

\section{References}

[1]AKYILDIZ, I., BRUNETTI, F., AND BLAZQUEZ, C. Nanonetworks: a new communication paradigm at molecular level. Computer Networks (Elsevier) 52 (August 2008), 2260-2279.

[2] Neus Roca Lacasa:Modeling Molecular Communication Networks. January 13, 2009

[3] FREITAS, R. J. Nanomedicine, Volume I: Basic Capabilities. Landes Biosience, 1999.

[4] Joan Capdevila Pujol : Bridging PHY and MAC Layers in Wireless Electromagnetic Nanonetworks. December 132010.

[5]M. Dragoman and D. Dragoman. Graphene based quantum electronics. Progress in Quantum Electronics, 33(6):165 - 214, 2009.

[6] Z.F. Wang, Huaixiu Zheng, Q.W. Shi, and Jie Chen. Emerging nanocircuit paradigm: Graphenebased electronics for nanoscale computing. In Nanoscale Architectures, 2007. NANOSARCH 2007. IEEE International Symposium on, pages 93 -100, 2007.

[7]Ossama Younis, Marwan Krunz, and Srinivasan Ramasubramanian : Node Clustering in Wireless Sensor Networks: Recent Developments and Deployment Challenges

[8] Ossama Younis and Sonia Fahmy : Distributed Clustering in Ad-hoc Sensor Networks: A Hybrid, Energy-Efficient Approach

[9] Ian F. Akyildiz And Josep Miquel Jornet, Georgia Institute Of Technology :Internet of Nano-things 